Electron Backscatter Diffraction in Low Vacuum Conditions

B. S. El-Dasher, S. G. Torres

July 22, 2008

Electron Backscatter Diffraction in Materials Science - 2 
This document was prepared as an account of work sponsored by an agency of the United States government. Neither the United States government nor Lawrence Livermore National Security, LLC, nor any of their employees makes any warranty, expressed or implied, or assumes any legal liability or responsibility for the accuracy, completeness, or usefulness of any information, apparatus, product, or process disclosed, or represents that its use would not infringe privately owned rights. Reference herein to any specific commercial product, process, or service by trade name, trademark, manufacturer, or otherwise does not necessarily constitute or imply its endorsement, recommendation, or favoring by the United States government or Lawrence Livermore National Security, LLC. The views and opinions of authors expressed herein do not necessarily state or reflect those of the United States government or Lawrence Livermore National Security, LLC, and shall not be used for advertising or product endorsement purposes. 


\title{
Electron Backscatter Diffraction in Low Vacuum Conditions
}

\author{
Bassem S. El-Dasher and Sharon G. Torres
}

Lawrence Livermore National Laboratory, Livermore, CA 94550 USA

\begin{abstract}
Most current scanning electron microscopes (SEMs) have the ability to analyze samples in a low vacuum mode, whereby a partial pressure of water vapor is introduced into the SEM chamber, allowing the characterization of nonconductive samples without any special preparation. Although the presence of water vapor in the chamber degrades electron backscatter diffraction (EBSD) patterns, the potential of this setup for EBSD characterization of nonconductive samples is immense. In this chapter we discuss the requirements, advantages and limitations of low vacuum EBSD (LV-EBSD), and present how this technique can be applied to a two-phase ceramic composite as well as hydrated biominerals as specific examples of when LV-EBSD can be invaluable.
\end{abstract}




\section{Introduction}

The ability to characterize specimens in a scanning electron microscope (SEM) at near atmospheric conditions has been around for some time (Lane 1970, Robinson 1974), with the first commercial systems pioneered by Danilatos and ElectroScan Corporation being released some 20 years ago (Danilatos 1981, 1983, 1985, 1990). Today, many commercial systems exist, and have become increasingly popular due to their ability to study insulating materials previously only observed in conventional SEMs after the application of a conductive coating.

Operation of such low vacuum systems (also called environmental SEMs or ESEMs) rely on the use of multiple pressure limiting apertures within the column, allowing the electron gun to maintain a high vacuum $\left(10^{-4} \mathrm{~Pa}\right)$ despite the chamber reaching pressures of up to $1 \mathrm{kPa}$. Chamber pressure control is by addition or removal of water vapor from a reservoir, and the presence of this vapor is what allows the direct imaging and analysis of insulators such as ceramics, polymers, and biological samples without the concern of charging typically associated with conventional "high vacuum" (SEMs) (Merideth 1996, Athene 2003, Donald 2003).

Figure 1 illustrates schematically the charge balance process that occurs within the chamber. Some electrons leaving the sample surface collide with water vapor molecules and in the process ionize them. The combination of positively charged detector and negatively charged sample surface drives the ions to the sample and upon contact, the ions balance out the charge. During the ionization process, daughter electrons are also knocked out, and some of these go on to interact with other vapor molecules and in doing so produce a cascade of electrons. The specialized gaseous detector is designed to attract these cascade electrons, allowing imaging of the sample surface. 


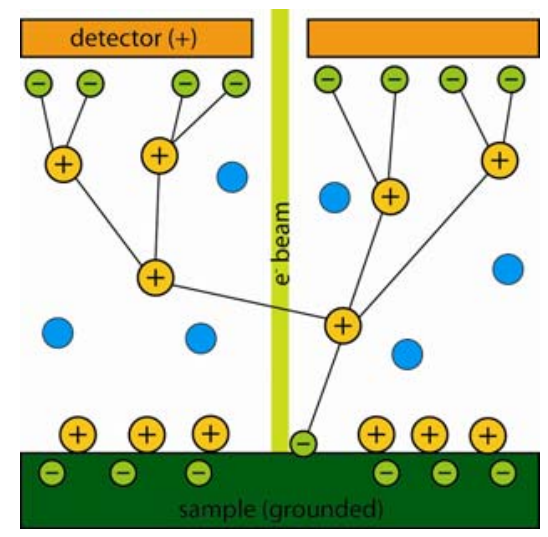

Figure 1. Schematic of the charge balance and imaging process in an ESEM.

The collisions that produce the cascade electrons naturally are of concern when considering low vacuum EBSD (LV-EBSD) analysis, as collisions of diffracted backscatter electrons with the water vapor degrade the diffraction pattern. Other tools that rely on characteristic radiation from the sample such as energy dispersive spectroscopy (EDS) are also affected, with decreased the effectiveness of quantitative analysis (Sigee 1998, Timofeeff et al 2000, Mansfield 2000). Nevertheless, LV-EBSD is a valuable tool, as illustrated by work such as that on minerals (e.g. Habesch 2000) and ceramic composites (e.g. Sztwiertnia et al 2006).

Through the use of LV-EBSD, studies on a variety of previously troublesome materials are now possible. In this chapter we identify the parameters important for successful LV-EBSD, and provide two distinct example applications to illustrate the approaches needed.

\section{Considerations for Low Vacuum EBSD}

While LV-EBSD is fundamentally similar to high vacuum (standard) EBSD, understanding how the chosen microscope parameters affects the quality of diffraction is important. In addition to the typical parameters of accelerating voltage and sample to detector distance, vapor pressure and dwell times become critical factors. In 
general, all the parameters are chosen based on the sample material, and the more nonconductive a material is, the lower the voltage, the shorter the dwell time or the higher the vapor pressure that is required. In practice, a combination of these parameters is what is employed, as too large a decrease in accelerating voltage or increase in vapor pressure would yield little to no diffraction.

As would be expected, the most significant affecter on diffraction in LV-EBSD is the presence of water molecules in the chamber. Although these molecules carry charge away from the sample surface and provide the ability to image nonconductive materials, they also absorb and/or deflect the backscattered electrons that make up the diffraction pattern. A demonstration of this effect is illustrated in Fig. 2, where the quality of diffraction patterns (measured as the average Hough peak intensity) collected from a silicon single crystal as a function of vapor pressure is plotted. A clear trend is observed, and the higher the vapor pressure, the lower the measured diffraction contrast. Fig. 3 shows diffraction patterns collected with a $20 \mathrm{kV}$



Figure 2. Plot of average diffraction pattern peak intensity as a function of vapor pressure. 

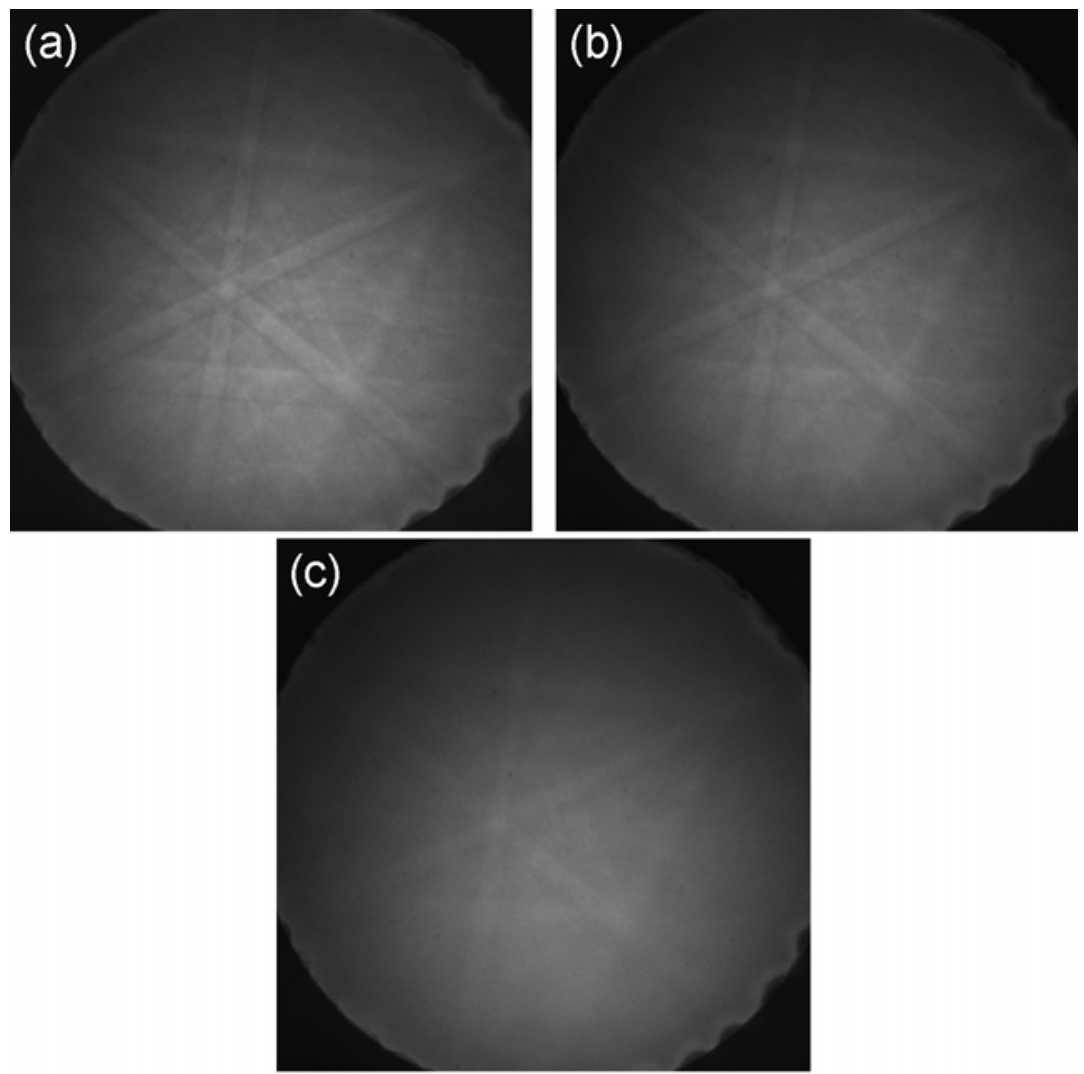

Figure 3. Raw diffraction patterns from a silicon single crystal at (a) $10^{-4} \mathrm{~Pa}$, (b) 50Pa, and (c) 200Pa.

source at three different pressures. A comparison between the high vacuum (Fig.3a) and the 50Pa (Fig. 3b) patterns shows that although some contrast is lost at $50 \mathrm{~Pa}$, the pattern is still quite clear and adequate for indexing. The 200Pa pattern (Fig. 3c), while recognizable, is significantly weaker in both intensity and contrast illustrating that the pressure needs to be limited in order to guarantee quality diffraction. 
During initial determination of parameters for a previously unexamined sample, it is suggested that the highest possible accelerating voltage be used. This allows for the largest possible diffraction volume, which is important for maximizing diffraction intensity of materials of interest such as oxides, nitrides and biominerals, all of which contain a significant amount of low atomic number elements. The general rules for EBSD hold however, and if the grain structure is too fine for the maximum accelerating voltage to be used due to overlapping diffraction patterns from neighboring grains, then a reduction in accelerating voltage is necessary.

Starting vapor pressure should be on the high end (e.g. 150Pa), and then reduced in increments to as low a pressure as possible without allowing charging to take place. Charging should be evaluated with the beam collimated (i.e. in spot mode), and using the diffraction pattern. If the pressure required to eliminate charging is too high for quality patterns, then the accelerating voltage and/or beam spot size need to be reduced. Although this has the undesirable side effect of decreasing diffraction contrast and hence requiring longer pattern collection times, it is necessary to iteratively adjust the parameters to achieve a balance between both beam and chamber parameters for a given sample material.

\section{Example Applications}

\section{Microstructural Analysis of AIN-TiB ${ }_{2}$ Ceramic Composite}

This example is intended to illustrate how despite the loss of signal cost associated with LV-EBSD, the technique can still be applied to one of the more challenging types of characterizations possible. We focus on $\mathrm{AlN}-\mathrm{TiB}_{2}$, a nonconductive ceramic composite (Sano 2006, 2006b), with the goal of collecting texture and grain distribution information without the use of a conductive carbon coating (since the samples were to undergo further surface characterization). Although this seems an ideal application of LV-EBSD, some difficulties arise due to the composition of the ceramic. 
First of these is the crystal structure of the constituents: they both are hexagonal. While possessing distinct structures, AlN is $\mathrm{P}_{3} \mathrm{mc}$ and $\mathrm{TiB}_{2}$ is $\mathrm{P} 6 / \mathrm{mmm}$ (see Fig. 4), many low index interplanar angles for both structures are the same, making distinguishing them using EBSD patterns error prone and inconsistent at best. This then requires the use of simultaneous energy dispersive spectroscopy (EDS) to identify the phase. For a more detailed description of phase identification approaches, please see CHAPTER XXXXX.

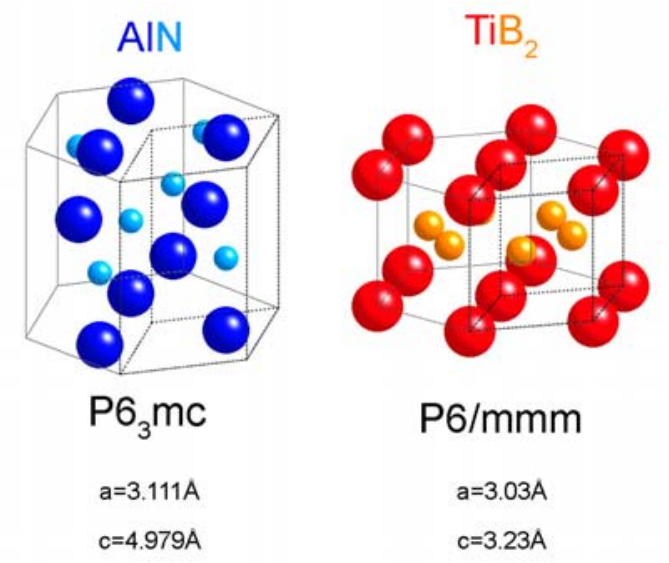

Figure 4. Crystal structures of $\mathrm{AIN}$ and $\mathrm{TiB}_{2}$.

As previously mentioned, the drawback to utilizing low vacuum mode is related to the water vapor, as it interferes with all characteristic radiation emitted from the sample, including X-rays. To gather sufficient signal to use EDS for phase discrimination, longer dwell times are needed (compared to diffraction collection), which causes local charging. In this specific example, as is likely for any example the reader may encounter, the correct settings were determined by trial and error. Microscope parameters (accelerating voltage and spot size) were chosen as were dwell time for EDS collection, and a scan was performed over a small region. The EBSD patterns collected were examined manually during the scan to determine if charging was occurring due to the chosen dwell time. If it was, the spot size, dwell time, and/or vapor pressure were then changed and the process 
repeated until no charging was detected. The ultimate goal was achieving the maximum signal possible while eliminating charging.
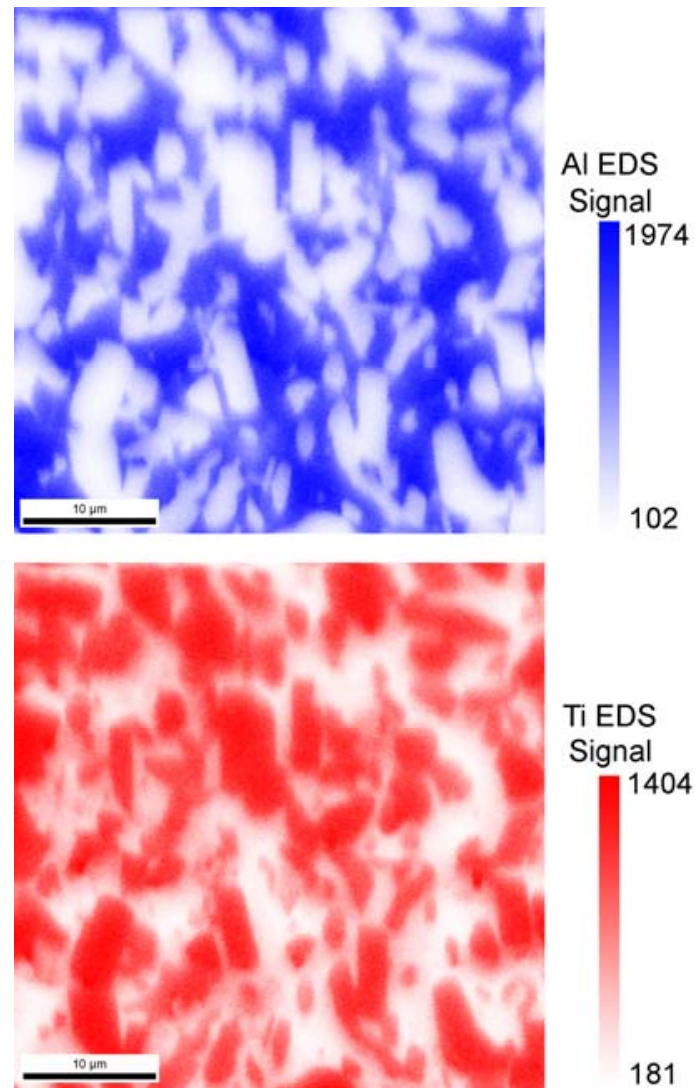

Figure 5. Energy dispersive spectroscopy signals for both $\mathrm{Al}$ and Ti used to distinguish between the two crystal types.

Once the parameters were set, scans were performed in the traditional manner. Fig. 5 shows EDS maps, illustrating the location of signals from both $\mathrm{Al}$ and Ti. This data, combined with the crystallographic information obtained from the EBSD patterns, can then yield a typical phase map as shown in Fig. 6.

This application then clearly illustrates that little needs to be given up when using LV-EBSD, as the capability of chemical discrimina- 


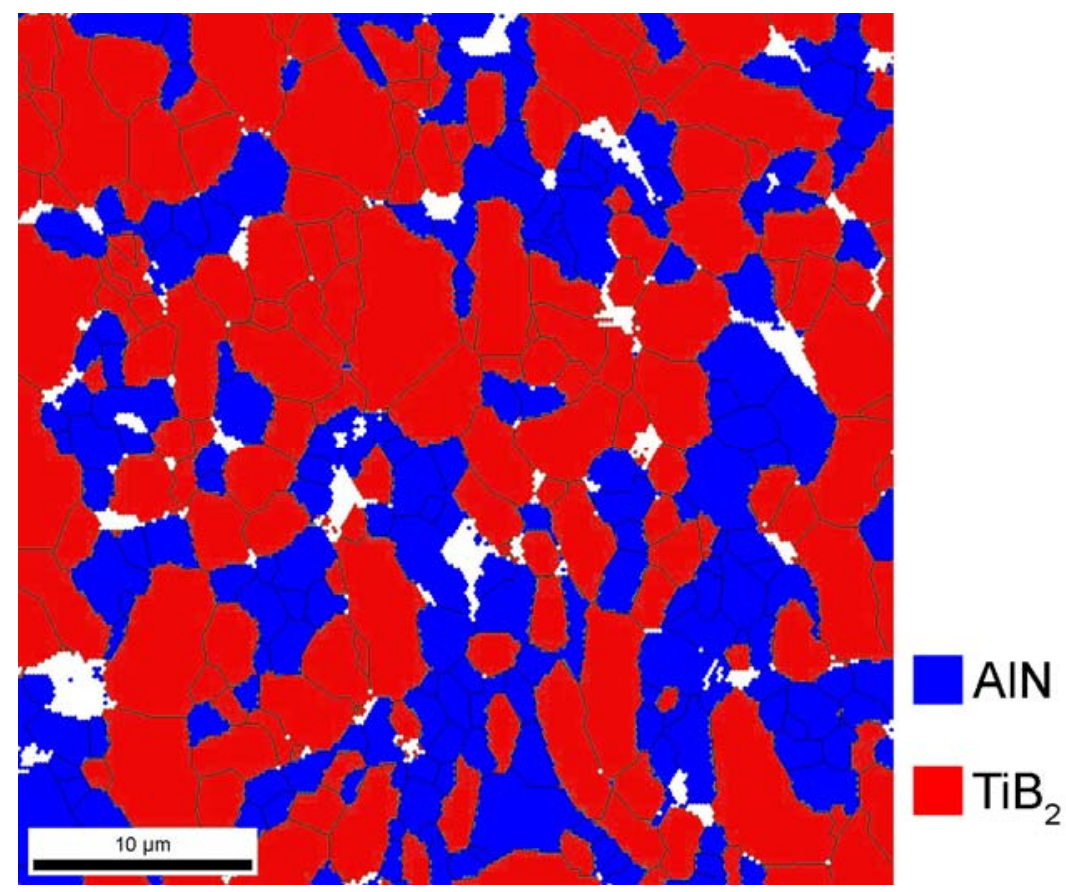

Figure 6. Phase map identifying the phase of individual grains.

tion using EDS can still be used if careful experimental parameter selection is used.

\section{Characterization of $\mathrm{CaHPO}_{4} \cdot 2 \mathrm{H}_{2} \mathrm{O}$ Single Crystals}

In this example, we deal with a somewhat different problem than that discussed in the preceding section. Typical applications of LVEBSD deal with nonconductive materials, but very rarely are these materials hydrated. Most, if not all, biomineral studies have been performed on dehydrated specimens, and this is due to the severe charging that occurs when collimated electrons are incident on water containing crystals.

The specific case discussed here considers a brushite $\left(\mathrm{CAHPO}_{4} .2 \mathrm{H} 2 \mathrm{O}\right)$ single crystal being used for crystal 
growth/dissolution experiments (Giocondi and Orme 2006). Surface studies showed faceted etch pits (Fig. 7), and determination of the crystallography of these pits was needed in order to properly model the behavior. While X-ray diffraction is typically used for this type of characterization, the size of the crystals (see Fig. 8) made it difficult to yield sufficient signal, making LV-EBSD potentially the sole alternative.

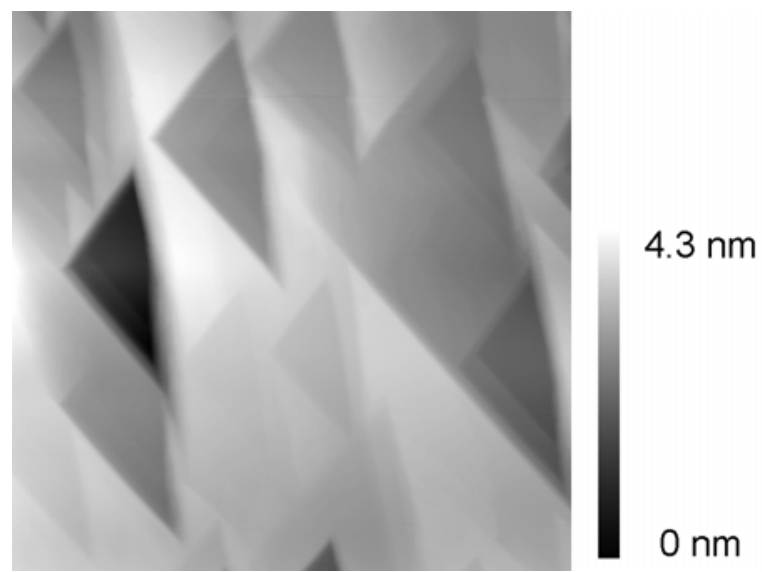

Figure 7. Atomic force microscopy surface topography micrograph showing etch pits on a brushite single crystal.

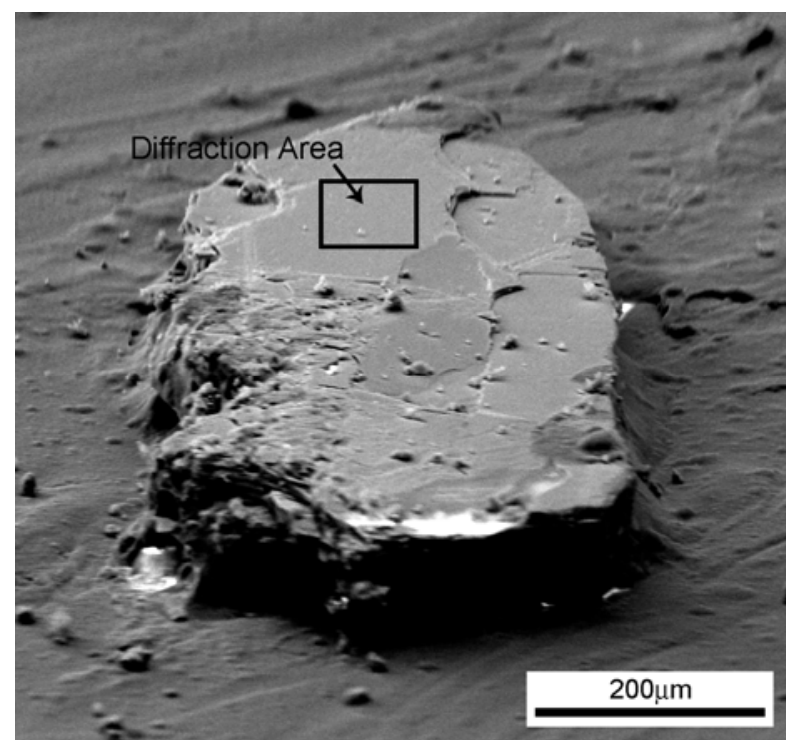

Figure 8. SEM micrograph of the brushite single crystal examined. 
With the severe charging concern in mind, it is imperative that the beam does not dwell on any area of the sample at any time. The first issue is the collection of the background signal. This was achieved by setting the microscope in scan mode and rastering the beam over an area that encompassed the whole sample at the fastest scan rate possible. While not ideal, this yielded a sufficiently accurate representation of the background. To collect the diffraction pattern, the beam was rastered at the highest scan rate again, although this time the area was limited to a small region on the crystal (shown in Fig. 8). This method was successful at yielding high-quality diffraction patterns, as shown in Fig. 9, and multiple patterns were collected from different areas of the crystal.
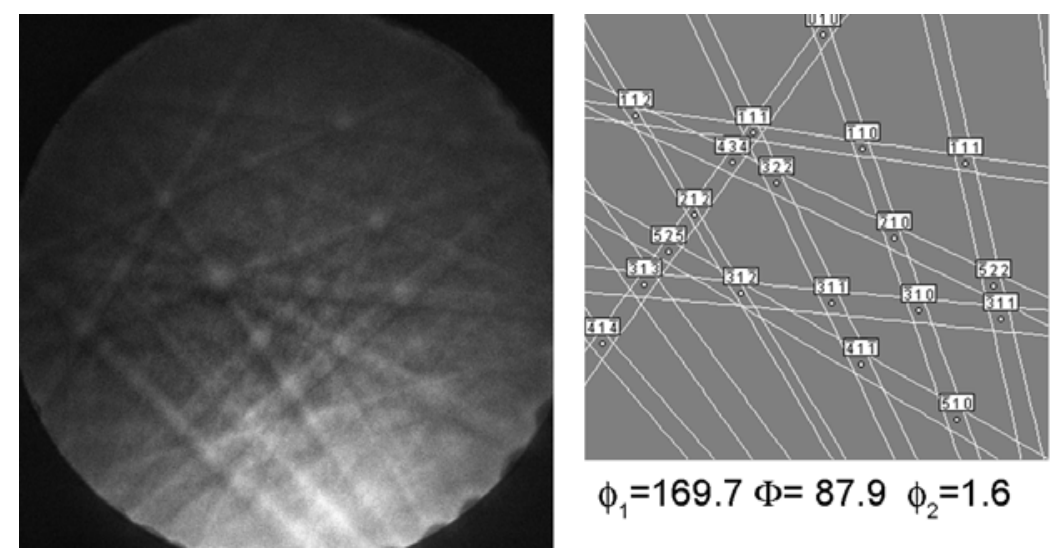

Figure 9. Diffraction pattern from brushite single crystal. The orientation determined from indexing the diffraction pattern was used to simulate diffraction for verification.

To index the diffraction patterns, two problems needed to be addressed first: how to accurately determine the pattern center, and which reflectors needed to be considered (no materials file for brushite exist). The pattern center was determined in a non-typical way: the exact working distance used in collecting the brushite diffraction patterns was noted, and the brushite crystal was removed and replaced with a silicon single crystal. The silicon crystal was then positioned at the exact same working distance and reference 
patterns were collected. These patterns were then used to determine the pattern center.

To determine the appropriate reflectors, the powder diffraction file (PDF) for brushite was obtained from the International Centre of Diffraction Data (ICDD) database, and the most intense reflectors were used. Although this was a problem as due to its low symmetry (monoclinic) there were a significant amount of relatively strong reflecting planes, knowledge of the cleavage planes was used. Since this crystal was cleaved, the surface being examined was either (010) or $(0 \overline{1} 0)$. By rotating simulated patterns about both plane normals', it was determined that the surface being examined was indeed (010), and comparison of the simulated patterns with the acquired pattern was used to iteratively down-select the reflectors, and the results of this analysis are shown in the simulated pattern in Fig. 9.

\section{ACKNOWLEDGEMENTS}

Part of this work was performed under the auspices of the U.S. Department of Energy by Lawrence Livermore National Laboratory in part under Contract W-7405-Eng-48 and in part under Contract DE-AC52-07NA27344. We gratefully acknowledge Jennifer Giocondi and Christine Orme at LLNL for the brushite single crystals, as well as Tomoko Sano and James Campbell of the Army Research Laboratory for the ceramic composite.

\section{REFERENCES}

Danilatos GD (1981) Design and construction of an atmospheric or environmental SEM (part 1). Scanning 4:9-20

Danilatos GD and Postle R (1983) Design and construction of an atmospheric or environmental SEM-2.Micron 14:41-52 
Danilatos GD (1985) Design and construction of an atmospheric or environmental SEM (part 3). Scanning 7:26-42

Danilatos GD (1990) Design and construction of an environmental SEM (part 4). Scanning 12:23-27

Donald AM (2003), The use of environmental scanning electron microscopy for imaging wet and insulating materials. Nature Materials 2:511-516

Giocondi JL and Orme CA (2006). Unpublished data

Habesch SM (2000) Electron backscattered diffraction analyses combined with environmental scanning electron microscopy: potential applications for non-conducting, uncoated mineralogical samples. Mat. Sci. Tech 16:1393-1398

Lane WC (1970) The environmental control stage. Scanning Electron Microsc.: 43-48

Mansfield JF (2000) X-ray analysis in the ESEM: a challenge or a contradiction. Mikrochimica Acta 132:137-143

Meredith P, Donald AM, and Thiel B (1996) Electron-gas interactions in the environmental SEM's gaseous detector. Scanning 18:467-473

Robinson VNE (1974) A wet stage modification to a scanning electron microscope. 8th Int. Congr. El. Microsc., Australian Academy of Science, Vol II:50-51

Sano T, Glide G, Campbell J et al (2006) Correlation Between Microstructure and Mechanical Properties for Hot Pressed TiB2-AlN Composites. Presentation at the 30th International Cocoa Beach Conference \& Exposition on Advanced Ceramics and Composites, January 22-27, 2006, Cocoa Beach, FL

Sano T, Campbell J, Glide G et al (2006b) Investigation of the Synergistic Effect on the Flexural Strength of TiB2-AlN by Microstructural Characterization. Poster at the Gordon Research Conference for Solid State Studies in Ceramics, August 13-17, 2006. Proctor Academy, Andover, $\mathrm{NH}$ 
Sigee DC (1998) Environmental SEM and X-ray microanalysis of biological materials. Mikrochimica Acta 15:283-293

Sztwiertnia K, Faryna M and Sawina G (2006) Misorientation characteristics of interphase boundaries in particulate $\mathrm{Al}_{2} \mathrm{O}_{3}$-based composites. J. Eur. Cer. Soc. 26:2973-2978

Timofeeff MN, Lowenstein TK and Blackburn WH (2000) ESEMEDS: an improved technique for major element chemical analysis of fluid inclusions. Chem.Geol. 164:171-182 